\title{
OBESITY-RELATED NEUTROPHILIA IN ENDOMETRIAL CANCER
}

\author{
M. Cheung a , D. Byrne ${ }^{b}$, O. O'Brien a , C. Le Roux c , T. Geoghegan 'b, D. Brennan a. \\ a Department of Gynecology, Mater Misericordiae University Hospital, Dublin, Ireland. \\ ${ }^{\mathrm{b}}$ Department of Radiology, Mater Misericordiae University Hospital, Dublin, Ireland. \\ ${ }^{\mathrm{c}}$ Conway Institute, University College Dublin, Dublin, Ireland.
}

\section{INTRODUCTION}

Obesity and metabolic syndrome are significant risk factors for the development of endometrial cancer (EC). One hypothesis is that increased visceral adiposity, which is associated with metabolic risk, drives inflammation and thus, oncogenesis. This case-control study aimed to measure differences in distribution of visceral and subcutaneous fat in endometrial cancer patients versus cervical cancer patients. We also compared distribution of pre-treatment white cells, between groups to see if there was a reliable biochemical marker that corresponded.

\section{METHODS}

Three patient groups were compared in this study, 22 patients with endometrial cancer (EC), 20 patients with cervical cancer (CC) and 20 patients with benign gynecological findings (BC) as controls. In order to eliminate selection bias in each group, 20 consecutive patients who presented to our institution in a 2 year period and underwent CT or PETCT imaging prior to treatment were selected. Body mass index (BMI) was calculated for each patient prior to treatment.

ImageJ software was used to measure the visceral fat percentage (VFP) on a L3/4 CT slice for each participant. VFP was calculated as a percentage of combined subcutaneous fat area (SFA) and visceral fat area (VFA) ((VFP = VFA/SFA+VFA)x100). BMI and VFP results for each group were compared using student's t-test analysis. Pre-treatment full blood counts were used to assess inflammatory ratios and compared between groups. Correlations were calculated using Pearson's correlation coefficient.

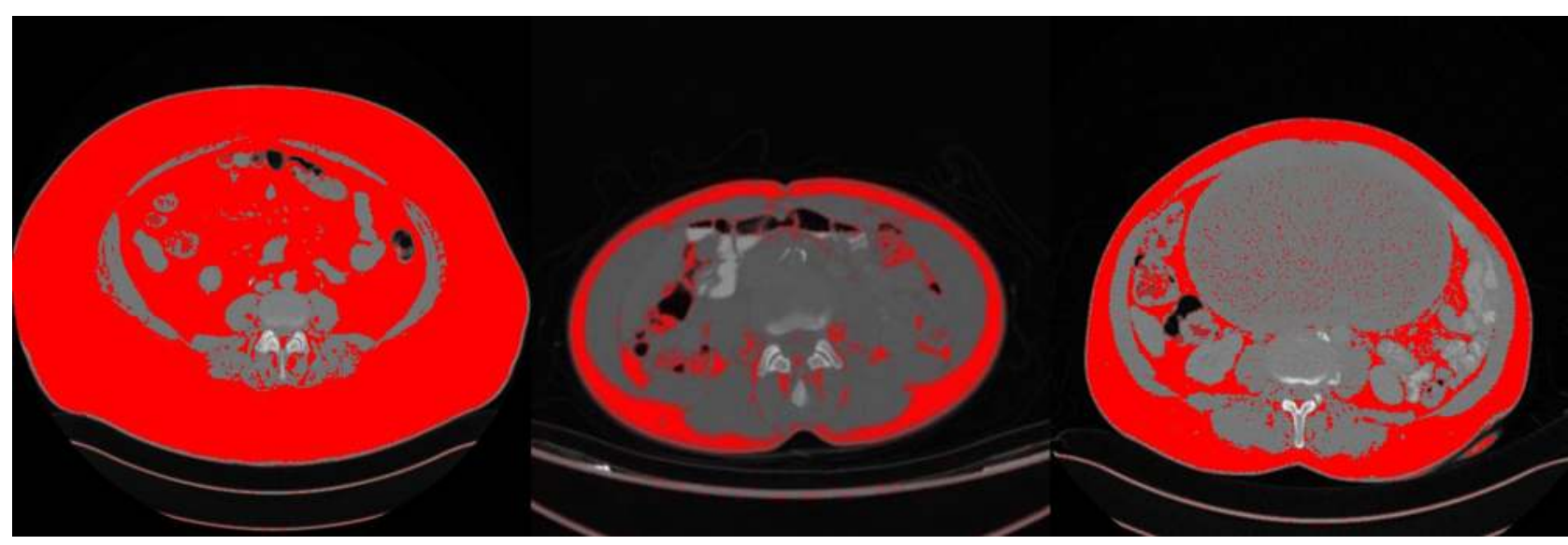

Fig. 1. Mean representation $L 3 / 4 C T$ slice for patient from endometrial cancer (EC), cervical cancer (CC) and benign control $(B C)$ groups. Red areas are highlighting tissue interpreted as adipose tissue.

\section{RESULTS}

Mean BMI (32.56 vs $25.87 \mathrm{~kg} / \mathrm{m} 2, \mathrm{p}=0.03$ ) and VFP (33.4 vs $24.0 \%, p=0.0018$ ) was higher in EC compared to CC patients. BMI did not correlate with VFP for EC $(r=0.076 ; p=0.74)$ or CC $(r=0.29 ; p=0.21)$ groups.

Mean pre-operative leukocytes (8.5 vs $7, p=0.044)$ and neutrophils (5.85 vs $4.32, p=0.019$ ) were higher in EC patients. There was no difference in the lymphocyte-neutrophil-ratio (LNR) or lymphocyte-monocyte-ratio (LMR), however the neutrophilmonocyte-ratio (NMR) was higher in the EC group (11.27 vs $8.28, p=0.018)$. This was due to the significant neutrophilia in the EC group (68.9\% vs $60.5 \%, p=0.037$ ) as there was no difference in monocytes in either group. No inflammatory ratio correlated with VFP.

Pre-op WBC \& neutrophils for EC \& CC groups

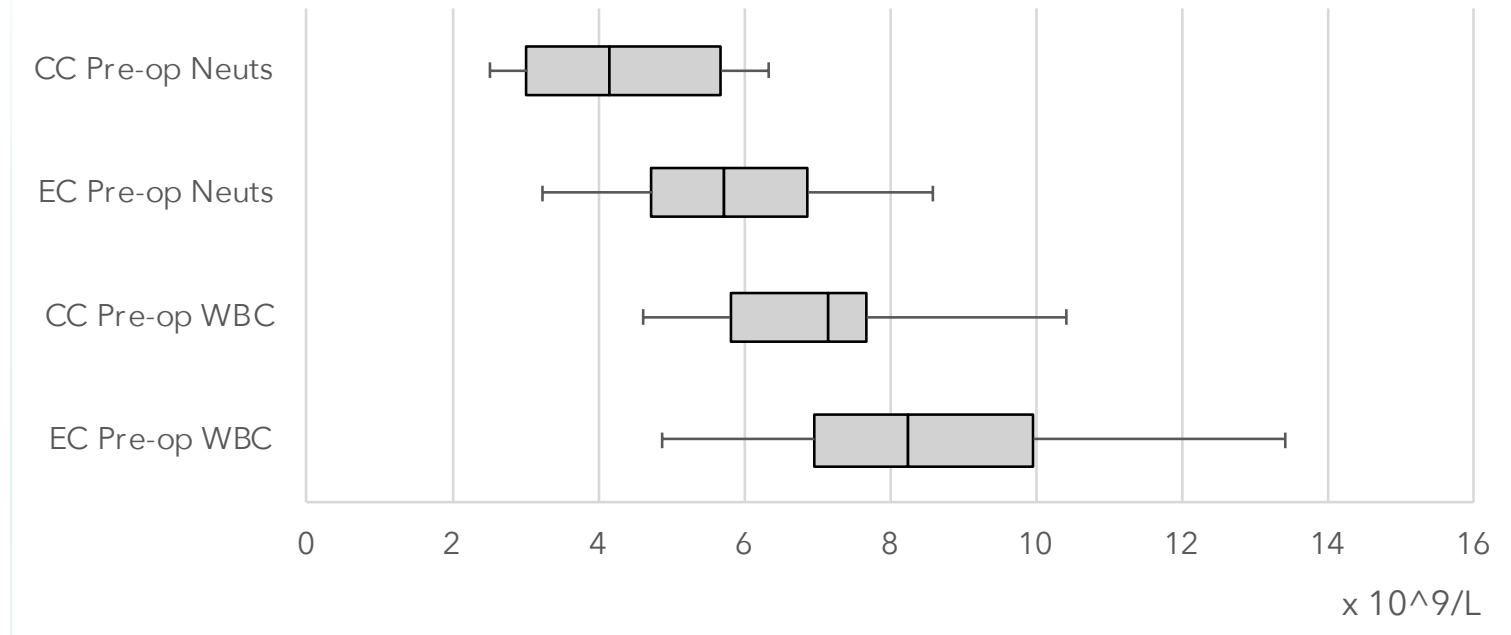

Fig. 2. Box plot showing mean pre-operative leukocytes (WBC) and neutrophils (Neuts) for endometrial cancer (EC) and cervical cancer (CC) groups.

\section{CONCLUSIONS}

This study shows that, as expected, EC patients have a higher BMI than CC patients. This also corresponds to a different body composition, with a greater proportion of their adiposity being visceral rather than subcutaneous. The relative neutrophilia seen in EC patients may represent a good biochemical marker for inflammation.

VFP and BMI do not correlate, and no white cell ratio correlated with either value in this study. Obesity related neutrophilia may be an important target in EC that warrants further investigation.

\section{References}

1. Zhang Y, Liu H, Yang S, Zhang J, Qian L, Chen X. Overweight, obesity and endometrial cancer risk: results from a systematic review and meta-analysis. Int J Biol Markers. 2014;29(1):e21-9.

2. Esposito K, Chiodini P, Capuano A, Bellastella G, Maiorino MI, Giugliano D. Metabolic syndrome and endometrial cancer: a metaanalysis. Endocrine. 2014;45(1):28-36.

3. Trabert B, Wentzensen N, Felix AS, Yang HP, Sherman ME, Brinton LA. Metabolic syndrome and risk of endometrial cancer in the united states: a study in the SEER-medicare linked database. Cancer Epidemiol Biomarkers Prev. 2015;24(1):261-7. 